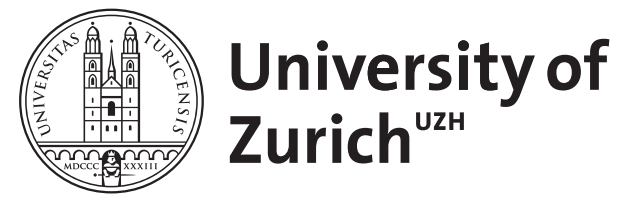

Experimental atrioventricular block without thoracotomy: a new instrument

Babotai, I ; Brownlee, R

\begin{abstract}
Experimental permanent total atrioventricular block was produced by a simplified method, without thoracotomy, by one person, and without the aid of x-ray apparatus. For this purpose, a newly designed cannula for the injection of $40 \%$ formalin into the bundle of His was made
\end{abstract}

DOI: https://doi.org/10.1093/cvr/5.3.416

Posted at the Zurich Open Repository and Archive, University of Zurich ZORA URL: https://doi.org/10.5167/uzh-155202

Journal Article

Published Version

Originally published at:

Babotai, I; Brownlee, R (1971). Experimental atrioventricular block without thoracotomy: a new instrument. Cardiovascular Research, 5(3):416-418.

DOI: https://doi.org/10.1093/cvr/5.3.416 


\title{
Experimental atrioventricular block without thoracotomy: a new instrument
}

\author{
I. Babotai and R. Brownlee ${ }^{1}$ \\ From Surgical Clinic A, University of Zürich, Switzerland
}

Authors' synopsis Experimental permanent total atrioventricular block was produced by a simplified method, without thoracotomy, by one person, and without the aid of $\mathrm{x}$-ray apparatus. For this purpose, a newly designed cannula for the injection of $40 \%$ formalin into the bundle of His was made.

In I 968 we reported in this journal a method for experimental production of total atrioventricular block (Turina, Babotai, and Wegmann, 1968). The method was based on the localization of the bundle of His with intracardial electrocardiogram monitoring by means of a special intravenous cannula. After bundle localization, $40 \%$ formalin was injected in the overlying myocardium, which resulted in a permanent total atrioventricular block. This previous method had several disadvantages. Firstly, fluoroscopy or image intensification apparatus was required, and two people were needed to operate this apparatus. Considerable practice and facility were required before the manipulation of the complicated injection system under radiographic control could be mastered. Other recently reported methods of experimental atrioventricular block production are even more complicated, in that they require thoracotomy, direct intracardiac visualization, fluoroscopy, or a combination of these procedures (Steiner and Kovalik, 1968; Scriabine, Rival, Bellet, Morgan, Weyn, and Nusbaum, 1969). The newly designed cannula eliminates these problems, since the block can be created without $x$-ray apparatus by one person.

${ }^{1}$ McLaughlin Travelling Fellow. University of Alberta Medical Center, Edmonton 61, Alberta, Canada.

\begin{abstract}
Method
I Apparatus

The newly designed cannula (Fig. I) is constructed of stainless steel tube $35 \mathrm{~cm}$ long and $3.5 \mathrm{~mm}$ in diameter. The outer cannula wall thickness is $0.5 \mathrm{~mm}$ and the inner cannula tip is $0.6 \mathrm{~mm}$ in diameter. The distal $\mathrm{I} \cdot 5 \mathrm{~cm}$ of both cannulas are curved at a $45^{\circ}$ angle. The curved end of the outer cannula has a half-round tip which acts as the intracardiac electrode and through which passes the inner needle. The entire outer cannula except for the exploring tip is electrically isolated with a coat of epoxy-resin. Attached to the proximal end of the outer cannula is a cable for ECG monitoring. To the proximal end of the inner cannula a connector for the formalin syringe is attached. The spring loaded inner cannula is held in the retracted position by a trigger. Pressure on the trigger releases the spring loaded inner cannula and thus permits it to drive the needle tip into the myocardium when the characteristic intracardiac bundle of His potential is found (see Fig. 2 and Technique). The length of inner cannula protrusion following trigger depression, and thus the depth of myocardial injection, is adjustable by a screw.
\end{abstract}

\section{Technique}

After induction of anaesthesia and tracheal intubation, the animal is laid left side down on the operating table. Under sterile conditions the right external jugular vein is dissected free. The cannula with the inner tip in the retracted position, and the lumen full of $40 \%$ formalin is then introduced into the right atrium via a venous cutdown. The direction of the cannula tip can then be controlled by means of the flange attached to 


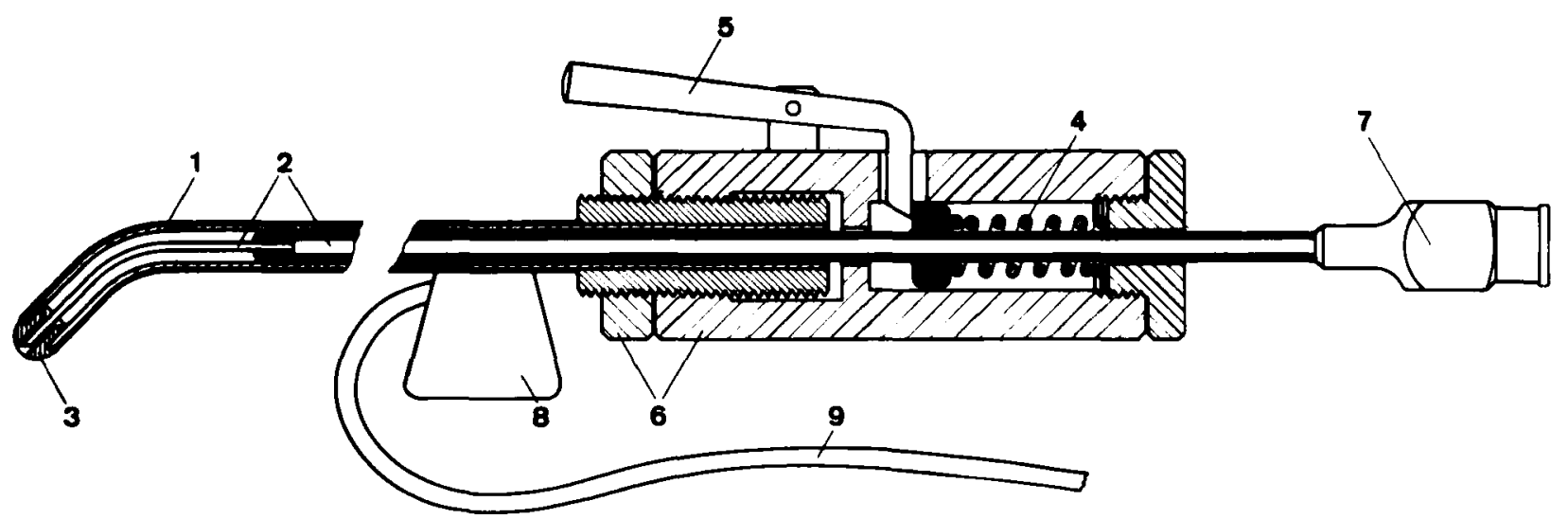

FIG. I Cannula for chronic AB block production. The inner cannula is in the retracted position. 1. Outer cannula with epoxy-resin isolation. 2. Inner cannula. 3. Intracardiac exploring electrode. 4. Spring. 5. Trigger. 6. Depth adjustment screw. 7. Luer-lock connector for formalin syringe. 8. Flange for cannula tip orientation. 9. ECG cable.

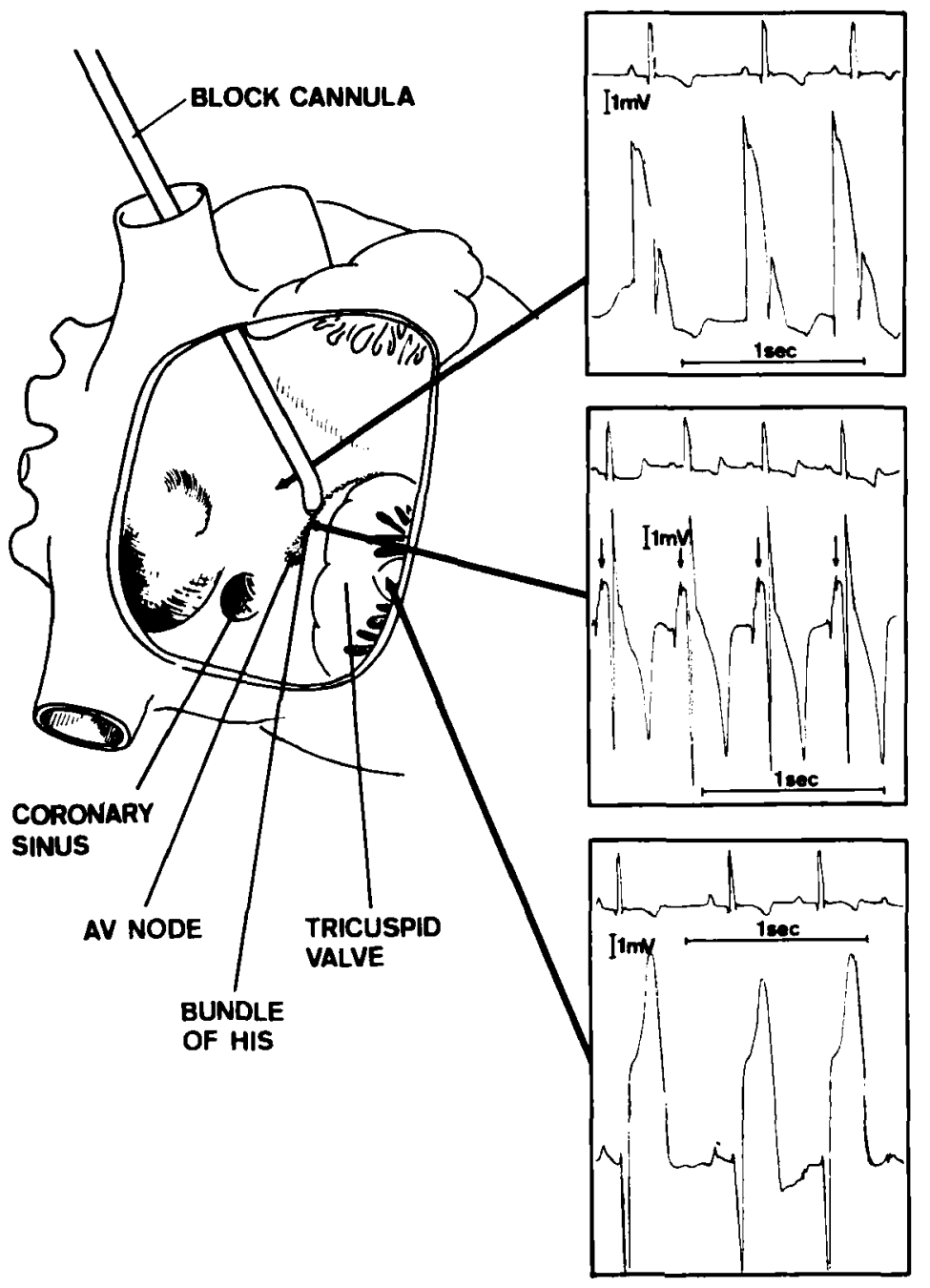

the distal end of the instrument. With the cannula connected to the ECG recorder, the intracardiac electrocardiogram is observed while the cannula tip is manipulated. When the typical potential for the bundle of $\mathrm{His}$ is found (Fig. 2), the cannula trigger is depressed, thus driving the spring loaded inner cannula 3 or $4 \mathrm{~mm}$ into the myocardium overlying the bundle. When movement of the cannula meets with resistance, good positioning of the needle tip in the myocardium is ensured. Formalin $0.4 \mathrm{ml}$. is then injected. When the cannula is in good position, some resistance to injection is encountered. Immediately a total AV block should appear. If no block is seen or a partial dissociation pattern appears, more formalin can be injected, since the cannula is held in good position by the inner needle in the myocardium. After creation of a total AV block, the inner cannula is pulled back, thus withdrawing the needle from within the myocardium, and the entire instrument is removed from the heart.

\section{Results}

The new instrument was used for block production in I I dogs. Total AV block was produced in to animals. One animal died before the block creation because of ventricular fibrillation after intracardiac manipulation of the cannula. Four dogs were killed $2 \mathrm{hr}$ after block creation. In six more dogs, pacemakers with intracardial electrodes were implanted after block production, in conjunction with another experiment.

Histological studies of the bundle of His

FIG. 2 A schematic representation of correct intracardiac cannula position before formalin injection and typical intracardiac Wilson electrocardiographic tracings. 


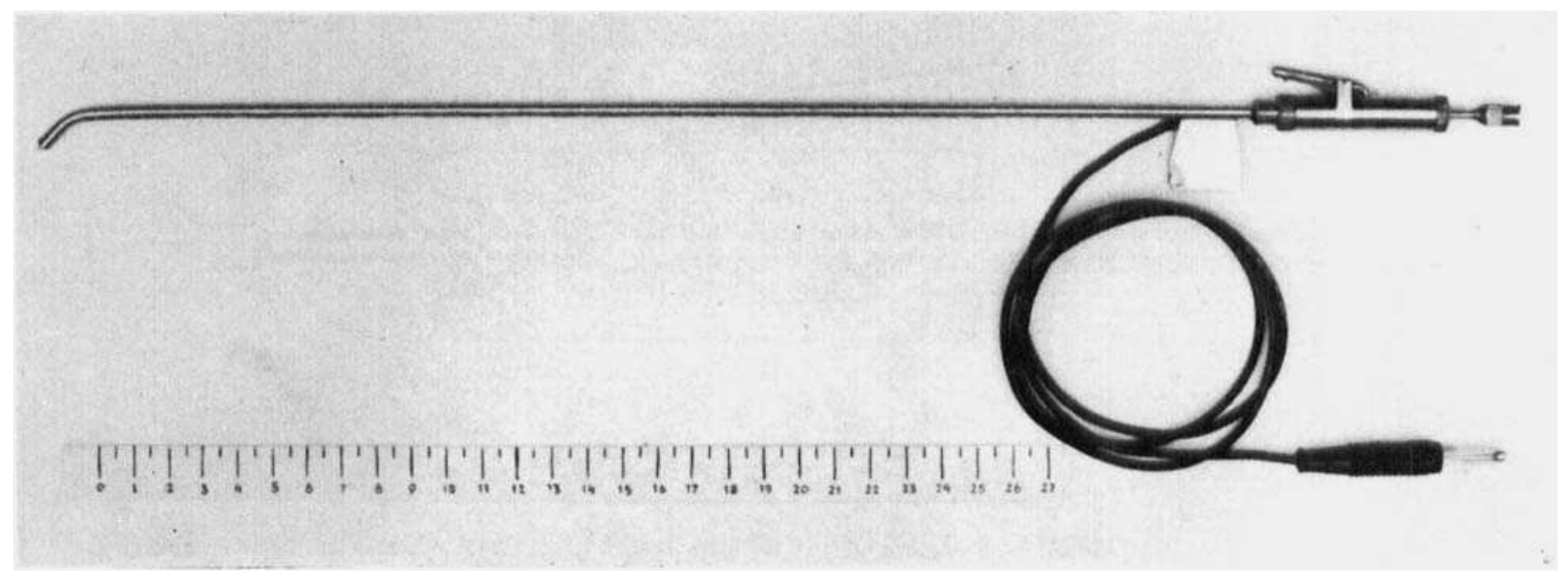

FIG. 3 Photograph of the newly designed cannula for production of experimental atrioventricular block without thoracotomy.

after injection of formalin have been previously reported by us, and are not included in this report (Turina and Babotai, 1967 ; Turina et al., 1968). The block became immediately apparent after injection and was permanent in each instance. No long-term complications, such as congestive heart failure or tachyarrhythmia, were encountered.

\section{Discussion}

Steiner and Kovalik (I968) have reported a relatively simple and reliable method of producing total AV block which involves formalin injection through the groove between the right atrium and aorta. This method eliminates the use of fluoroscopy but necessitates a right thoracotomy. Scriabine et al. (1969), in studying drug effects on ventricular rate in total AV block, ligated the bundle of His under direct vision. Despite the greater magnitude of the operation, successful block was produced in only about $70 \%$ of animals.

The new instrument described above is an improvement on these previously reported methods, in that a chronic atrioventricular block can be created in animals without the necessity of $x$-ray apparatus. When the direct-writing electrocardiogram is in close proximity to the operator, allowing ease of intracardiac electrocardiogram monitoring, the block can be produced by one person.

The spring loaded cannula needle tip eliminates the problem of loss of correct positioning of the cannula tip during manipulation before formalin injection. Also, the needle goes securely into myocardium superficial to the bundle, so that the possibility of injecting formalin into the blood stream or outside the heart is diminished. The positioning of the needle tip in the myocardium can be tested by the movement of the entire cannula. When the needle is in the myocardium, resistance to movement of the cannula is met. Because the entire instrument tip is fabricated from metal, the intracardiac electrocardiogram can be monitored directly during formalin injection. This feature allows an additional assurance that the formalin injection has been properly placed.

\section{References}

Turina, M., and Babotai, I. (1967). Erzeugung eines totalen atrioventrikulären Blocks beim Hund ohne Thorakotomie. Thoraxchirurgie,, 15, 601608.

Turina, M., Babotai, I, and Wegmann, W. (I968). Production of chronic atrioventricular block in dogs without thoracotomy. Cardiovascular $R e$ search, 2, 389-393.

Steiner, C., and Kovalik, A. T. W. (1968). A simple technique for production of chronic complete heart block in dogs. Journal of Applied Physiology, 25, 631-632.

Scriabine, A., Rival, J., Bellet, S., Morgan, G. Weyn, A. S., and Nusbaum, M. (I 969). Effect of protokylol on the ventricular rate in dogs with experimental A-V heart block. American Hear Journal, 77, 649-652. 